\title{
Philosophiques
}

\section{Note sur l'identité intentionnelle}

\section{Richard Vallée}

Volume 26, numéro 1, printemps 1999

URI : https://id.erudit.org/iderudit/004993ar

DOI : https://doi.org/10.7202/004993ar

Aller au sommaire du numéro

Éditeur(s)

Société de philosophie du Québec

ISSN

0316-2923 (imprimé)

1492-1391 (numérique)

Découvrir la revue

\section{Citer cet article}

Vallée, R. (1999). Note sur l'identité intentionnelle. Philosophiques, 26(1), 37-52. https://doi.org/10.7202/004993ar

\section{Résumé de l'article}

L'identité intentionnelle soulève des énigmes quant au comportement linguistique de certains pronoms anaphoriquement liés à des quantificateurs restreints, mais qui ne peuvent être considérés comme des variables liées par ces quantificateurs. J'examine ce problème et suggère une solution, qui est une généralisation de la notion de pronom de texte (Vallée, 1994), conçue afin d'analyser certains pronoms qui ont un comportement linguistique similaire. d'utilisation que vous pouvez consulter en ligne.

https://apropos.erudit.org/fr/usagers/politique-dutilisation/ 


\title{
Note sur l'identité intentionnelle
}

\author{
RICHARD VALLÉE \\ rvallee@gpu.sru.ulberta.ca \\ University of Alberta
}

RÉSUMÉ. - L'identité intentionnelle soulève des énigmes quant au comportement linguistique de certains pronoms anaphoriquement liés à des quantificateurs restreints, mais qui ne peuvent être considérés comme des variables liées par ces quantificateurs. J'examine ce problème et suggère une solution, qui est une généralisation de la notion de pronom de texte (Vallée, 1994), conçue afin d'analyser certains pronoms qui ont un comportement linguistique similaire.

ABSTRACT. - Intentional identity raises problems with respect to the linguistic behavior of anaphoric pronouns linked to restricted quantifiers but that cannot be variables bound by these quantifiers. I examine this issue and suggest a solution based on the notion of a text pronoun (Vallée, 1994), introduced to deal with pronouns exhibiting a similar linguistic behavior.

\section{Introduction}

\section{Considérons}

(1) Marie croit qu'un fantôme hante la maison de Jean

L'analyse de la description indéfinie "un fantôme » à l'aide d'un quantificateur, d'un prédicat et de variables (par « $(\exists x)$ (fantôme $x)$ ) ) ou, dans une notation plus contemporaine, en termes de quantificateur restreint (par «[un $\mathrm{x}$ : fantôme $\mathrm{x}]$ »), représente une innovation majeure en philosophie du langage'. Elle permet de mettre au jour l'ambiguïté syntaxique de (1) en plaçant le quantificateur restreint, "un fantôme ", soit avant, soit après le terme d'attitude propositionnelle, pour obtenir

(2) [Un x: fantôme $\mathrm{x}]$ Marie croit que (x hante la maison de Jean)

ou

(3) Marie croit que ([un x: fantôme $x]$ (x hante la maison de Jean))

En (2), le quantificateur restreint a une portée large et constitue un exemple de quantification à l'intérieur — le quantificateur restreint se situe à l'extérieur du contexte d'attitude propositionnelle et lie une variable qui se trouve à l'intérieur de celui-ci - bien connu pour soulever des problèmes depuis les années $50^{2}$. En (3), le quantificateur restreint a une portée étroite : il se situe à l'intérieur du

I. J'utiliserai, dans le présent texte, des quantificateurs restreints. Un quantificateur restreint contient, dans une première clause, un quantificateur liant une variable et, dans une seconde, le domaine de cette variable est restreint par un terme général. Ainsi, "plusieurs hommes " devient «plusieurs $\mathrm{x}$ : homme $\mathrm{x}$ ».

2. Voir Quine, W.v.O., Word and Object, The MIT Press, 1960. 
contexte d'attitude propositionnelle et lie une variable qui s'y trouve également. L'ambiguité syntaxique de (1), mise au jour par (2) et (3), trouve un écho au niveau sémantique. (2) et (3) explicitent deux interprétations sémantiques de (1). Si la phrase (2) est vraie, alors il existe un fantôme et Marie croit qu'il hante la maison de Jean ; la vérité de la phrase (3) n'implique pas l'existence de fantômes : elle est vraie si et seulement si Marie croit le contenu conceptuel exprimé par ce qui suit le terme d'attitude propositionnelle. Je dirai que (2) est une attribution de croyance objectuelle - elle requiert un objet, en l'occurrence un fantôme, pour être vraie - et que (3) est une attribution de croyance conceptuelle - elle ne requiert pas l'existence d'objets pour être vraie.

L'identité intentionnelle ${ }^{3}$ soulève des problèmes majeurs lorsqu'il s'agit de donner la forme logique de certains énoncés d'attitude propositionnelle qui doivent être conçus comme des attributions de croyance conceptuelle. Ces difficultés ne concernent pas directement la célèbre théorie russellienne des descriptions définies 4 mais l'analyse de "un fantôme ", ou de "quelques fantômes ", en termes de quantificateurs restreints. Elle atteint cependant la première en minant la dernière, puisqu'elle fait apparaître les limites de notre approche de certaines expressions comme quantificateurs restreints. Les deux premières parties de mon texte sont consacrées à une présentation de ces problèmes. L'identité intentionnelle semble exiger qu'un quantificateur restreint ayant une portée étroite dans un premier énoncé d'attitude propositionnelle lie une variable qui se trouve dans une phrase enchâssée dans un second énoncé d'attitude propositionnelle, lequel doit être interprété comme une attribution de croyance conceptuelle ${ }^{5}$. Ce type de quantification, connu sous le nom de quantification à l'extérieur, ne peut que laisser perplexe. Dans la troisième partie de mon texte, je soutiendrai qu'il n'y a jamais de quantification à l'extérieur. Le pronom que nous voulons traiter comme variable liée ne se soumet pas à cette analyse, et il doit être considéré comme ce que j'ai déjà appelé un pronom de texte ${ }^{6}$.

\section{Les pronoms comme variables liées.}

Les pronoms à la troisième personne du singulier (« il »/« elle ») ou du pluriel («ils»/« elles») sont parfois des termes référentiels, dit démonstratifs, exigeant une démonstration pour référer à un objet. Par exemple, dans «Il

3. Voir Geach, P., «Intentional Identity ", Journal of Philosophy 64, 1967, p. 627-632. Aussi dans Geach, P., Logic Matters, 1972, Blackwell, Oxford, p. 146-153.

4. Voir Russell, B., "On Denoting, Mind", 1905. Aussi dans Russell, B. Logic and Knowledge, London, Routledge and Kegan Paul, 1956, p. 41-56.

5. Voir Geach, P., "Intentional Identity », p. 630.

6. Voir Vallée, R., "Descriptions, référence et anaphore », Dialogue 33, 1994, p. 611-634.

7. Voir Kaplan, D., « Demonstratives ", dans Perry, J., J. Almog et H.Wettstein (dir. de publication), Themes from Kaplan, New York University Press, 1989, p. 481-563, et Vallée, R, "Who are We »? Canadian Journal of Philosophy 26, n 2, 1996, p. 211-230. 
est gros », le pronom demande une démonstration pour désigner un objet, et peut désigner différents objets lors de différentes énonciations. Si je dis «Il est gros » en montrant un chat, "il » réfère à ce chat. Si je prononce la même phrase en pointant vers un autre chat, «il » désigne cet autre chat. Les démonstratifs sont sensibles au contexte extralinguistique de l'énonciation. Les pronoms à la troisième personne réfèrent parfois par l'intermédiaire d'une expression référentielle, de laquelle ils sont donc sémantiquement dépendants. Une expression dont la valeur sémantique dépend de celle d'une autre, appelée son antécédent, est dite anaphoriquement liée à cette autre. Nous dirons qu'elle est anaphoriquement liée à son antécédent. Ainsi, dans une certaine interprétation de "Pierre a dit qu'il viendrait ", " il " réfère à Pierre, mais exerce ce rôle par le biais du terme référentiel " Pierre ", auquel le pronom est donc anaphoriquement lié et qui est son antécédent ${ }^{8}$. Le pronom «il » est alors sensible au contexte intralinguistique de l'énonciation et coréférentiel avec "Pierre ». Les pronoms sont d'une grande souplesse. Dans une certaine interprétation de

(4) Chaque homme croit qu'il est mortel

le pronom «il » n'est ni démonstratif ni anaphoriquement lié à un terme référentiel. Il entretient cependant une relation évidente avec " chaque homme ", et sa valeur sémantique semble dépendre de cette dernière expression. $\mathrm{Si}$ " chaque homme » est rendu par " chaque $\mathrm{x}$ : homme $\mathrm{x}$ ", le pronom peut être traité comme une variable liée par le quantificateur restreint. On obtient alors la forme logique

$$
\text { [Chaque } \mathrm{x} \text { : homme } \mathrm{x} \text { ] ( } \mathrm{x} \text { croit que ( } \mathrm{x} \text { est mortel)) }
$$

On note plus rarement que les pronoms à la troisième personne ayant une occurrence dans une description, définie ou indéfinie, sont aussi parfois des variables liées. Il en va ainsi de " il » dans la description définie « la femme qu'il aime ", comme le montre la forme de

(6) Chaque homme a confiance en la femme qu'il aime

soit

(7) [Chaque $\mathrm{x}$ : homme $\mathrm{x}]$ ([la $\mathrm{y}$ : femme $\mathrm{y} \cdot \mathrm{x}$ aime $\mathrm{y}](\mathrm{x}$ a confiance en y))

Le traitement des descriptions (définies et indéfinies) et, plus généralement, des termes quantifiés (comme " chaque homme ", " tous les hommes", " certains hommes ", etc.) en termes de quantificateurs restreints, en plus de ses qualités propres, permet de rendre compte du fonctionnement sémantique de certains pronoms, lesquels sont des variables liées. Ces traits ont convaincu plusieurs du bien-fondé de cette analyse. En effet, la forme logique de ces termes permet non seulement de donner les conditions de vérité des phrases dans lesquelles ils ont une occurrence, mais aussi d'expliciter des relations intrasentencielles entre des éléments lexicaux, dans ce qui constitue un

8. Une expression anaphorique n'a qu'un antécédent. 
élégant traitement de certains pronoms. Malheureusement, une telle approche se heurte à l'identité intentionnelle.

\section{L'identité intentionnelle}

Certaines phrases d'attitude propositionnelle contiennent une description indéfinie ayant une occurrence dans la phrase succédant au verbe d'attitude propositionnelle, par exemple " un fantôme » dans (1). Comme nous l'avons vu, cette phrase peut recevoir deux interprétations sémantiques - elle peut constituer une attribution de croyance objectuelle ou une attribution de croyance conceptuelle - , lesquelles sont saisies syntaxiquement en faisant varier la portée du quantificateur restreint, pour obtenir soit (2), soit (3). Prenons maintenant la phrase complexe

(8) Marie croit qu'un fantôme hante la maison de Jean et Pierre croit qu'il a brisé des carreaux

où la valeur sémantique de "il » dépend manifestement de celle de " un fantôme ", terme avec lequel le pronom est donc anaphoriquement lié. Puisque " un fantôme » est un quantificateur restreint, "il » devrait être traité comme une variable liée. C'est ici que surgit le problème de l'identité intentionnelle. (8) peut être interprétée comme l'attribution à Marie d'une croyance purement conceptuelle, qui n'engage pas le locuteur à l'existence d'un fantôme et qui n'exige pas l'existence de fantôme pour être vraie (le quantificateur restreint a donc une portée étroite), et l'attribution à Pierre d'une croyance aussi purement conceptuelle, n'engageant pas le locuteur à l'existence d'un fantôme et n'exigeant pas l'existence de fantômes pour être vraie. Geach (1967) note que, dans cette interprétation, intuitivement, le fantôme que Pierre croit avoir brisé des carreaux est « le même » que celui que Marie croit hanter la maison de Jean. Il réécrirait alors, comme il le fait souvent dans ses exemples, "Marie croit qu'un fantôme hante la maison de Jean et Pierre croit qu'il (le même fantôme) a brisé des carreaux. » Cette interprétation de (8) est tout à fait naturelle. Comme le résume Geach,

We have intentional identity when a number of people, or one person on different occasions, have attitudes with a common focus, whether or not there is actually something at the focus ${ }^{9}$.

La formulation utilisée par Geach afin de clarifier son intuition ( "Marie croit qu'un fantôme hante la maison de Jean et Pierre croit qu'il (le même fantôme) a brisé des carreaux ») est un peu trompeuse, semblant impliquer que les croyances attribuées concernent un seul et même fantôme. Il devrait être clair que le problème qui nous occupe vient justement du fait qu'il n'y a aucun fantôme dont on puisse dire qu'il est l'objet de la croyance de Marie et de celle

9. Geach, P., «Intentional Identity », p. 627. 
de Pierre. L'expression « le même » vient simplement expliciter l'intuition que les deux personnes se voient attribuer des croyances ayant un même foyer, et non qu'il existe un unique objet vers lequel convergent effectivement leurs pensées. Comment rendre compte de l'intuition selon laquelle on peut attribuer, à deux personnes, des croyances conceptuelles ayant le même foyer ${ }^{10}$ ? Il est évident que, si on veut respecter notre intuition sémantique que Pierre a une croyance conceptuelle ayant le même foyer que celle de Marie, les deux croyances doivent avoir un contenu similaire, et on ne peut se dispenser de recourir à la relation d'anaphore afin de saisir cette similarité. En vertu de cette relation, le contenu de croyance attribué à Pierre, en utilisant un pronom anaphorique, est fonction du quantificateur restreint antécédent, puisque la valeur sémantique de ce pronom en dépend. Si ce quantificateur restreint donne, partiellement, la croyance de Marie, alors la croyance attribuée à Pierre est fonction, partiellement, de celle attribuée à Marie. Si la croyance attribuée à Pierre est similaire à celle attribuée à Marie, alors notre intuition syntaxique d'un lien anaphorique est fondée. Enfin, on ne voit pas très bien comment on pourrait respecter notre intuition syntaxique d'un lien anaphorique entre " un fantôme » et «il », et nier que le contenu de croyance attribué à Pierre est similaire à celui attribué à Marie.

La seconde phrase de (8), comme attribution de croyance conceptuelle, peut signifier, dans une première approximation, que Pierre croit que le fantôme que Marie croit hanter la maison de Jean a brisé des carreaux ". On conviendra toutefois que l'attribution d'un tel contenu de croyance ne soit pas toujours plausible. Dans les cas intéressants, elle ne l'est pas. La croyance attribuée à Pierre ne dépend pas forcément du fait que Marie croie qu'un fantôme hante la maison de Jean. Elle dépend, au mieux, du matériel linguistique utilisé pour attribuer un contenu de croyance à Marie. D'ailleurs, Pierre peut parfaitement se voir attribuer une croyance purement conceptuelle, par la seconde phrase de (8), tout en ignorant ce que croit Marie, ce qui est impossible selon le contenu explicité ci-dessus. Supposons que Marie ait la croyance, conceptuelle, qu'un fantôme hante la maison de Jean. Supposons aussi que Pierre, qui ne connaît pas Marie, lise dans un journal qu'un fantôme hante la maison de Jean et en vienne à penser qu'il a brisé des carreaux. Pierre ne connaît pas les croyances de Marie et ne sait pas non plus que Marie existe. L'énonciation de (8), dans l'interprétation qui nous intéresse, serait cependant non seulement acceptable mais aussi vraie. Malheureusement, l'expression le fantôme que Marie croit hanter la maison de Jean, utilisée pour relater le contenu de croyance de Pierre, implique que ce dernier a une croyance quant aux croyances de Marie. Enfin, dans cette interprétation de la seconde phrase de (8), le pronom n'est pas, à strictement parler, anaphoriquement lié au

Io. On peut avoir le même problème en considérant deux attributions différentes à un même agent, comme dans " Marie croit qu'un fantôme hante la maison de Jean. Elle croit aussi qu'il a brisé des carreaux" .

I I. Voir Saarinen, E., « Intentional Identity and Interpretation » : A Case Study of the Relations Among Quantifiers, Pronouns and Propositional Attitudes ", Linguistics and Philosophy 2, 1978, p. 151-223. 
quantificateur restreint qui est son antécédent, puisque la contribution sémantique de ce pronom va bien au-delà de ce que fournit ce quantificateur restreint. Malheureusement, le mécanisme par lequel le pronom peut apporter une telle contribution sémantique n'est pas explicité. Je ne tiendrai pas compte de l'interprétation de la seconde phrase de (8) suggérée par Saarinen. Je reviendrai cependant sur cette interprétation à la dernière section de mon texte. Mais quelle est la contribution sémantique de « il » dans (8)? Quelle est la croyance attribuée à Pierre?

L'intuition sémantique que Marie et Pierre se voient attribuer des croyances conceptuelles ayant le même foyer ne peut malheureusement être saisie, syntaxiquement, en traitant le pronom comme une variable liée. Dans la mesure où une croyance conceptuelle est attribuée à Marie et à Pierre, on peut d'office refuser de placer le quantificateur restreint en portée large et d'accorder à (8) la forme " [un x : fantôme x] (Marie croit que (x hante la maison de Jean) et Pierre croit que (x a brisé des carreaux)) ". Accorder une portée étroite au quantificateur restreint " un fantôme ", et représenter (8) par

(9) Marie croit que ([un $\mathrm{x}$ : fantôme $\mathrm{x}$ ] (x hante la maison de Jean et Pierre croit que $\mathrm{x}$ a brisé des carreaux))

ne donne pas non plus le résultat escompté. (9) attribue à Marie le contenu constitué par tout ce qui suit la première occurrence de «croit que » - « un fantôme hante la maison de Jean et Pierre croit qu'il a brisé des carreaux ». Mais ce n'est pas l'interprétation visée. On voudrait que le « et » marque la frontière entre deux phrases, que chacune attribue une croyance conceptuelle respectivement à Marie et à Pierre, et que " un $\mathrm{x}$ : fantôme $\mathrm{x}$ » soit l'antécédent $\mathrm{du}$ « il » se trouvant dans la seconde. On aurait alors

(10) Marie croit que ([un $\mathrm{x}$ : fantôme $\mathrm{x}]$ ( $\mathrm{x}$ hante la maison de Jean)) et Pierre croit que ( $\mathrm{x}$ a brisé des carreaux)

Malheureusement, on ne peut obtenir un tel résultat. Il y a deux obstacles entre le quantificateur restreint et le pronom.

Dans l'interprétation des phrases de (8) comme attributions de croyance purement conceptuelle, chaque clause indirecte de (8) est syntaxiquement isolée, avec pour effet que le quantificateur restreint de la première ne peut rejoindre le "il " de la seconde ${ }^{12}$. La barrière de la phrase est un second obstacle. Il est facile de rendre manifeste la présence de deux phrases si on sépare (8) dans la séquence suivante, où $\mathrm{A}$ et $\mathrm{B}$ sont deux locuteurs différents :

A. Marie croit qu'un fantôme hante la maison de Jean

B. Pierre croit qu'il a brisé des carreaux

On constate alors que, même en portée large, le quantificateur restreint « un fantôme " ne peut atteindre le «il » de la seconde phrase de (8), puisque le 
quantificateur ne peut traverser la frontière de la première phrase pour lier un terme qui se trouve dans la seconde ${ }^{13}$.

Il va sans dire que « il » ne peut être un simple pronom de paresse ${ }^{14}$. (8) serait alors interprétée par «Marie croit qu'un fantôme hante la maison de Jean et Pierre croit qu'un fantôme a brisé des carreaux ", où non seulement les croyances peuvent porter sur des objets différents, mais où l'usage de telles phrases laisse aussi entendre qu'il s'agit de deux fantômes différents ${ }^{15}$. Le problème a deux aspects. Le premier, syntaxique, concerne la forme logique de la seconde phrase de (8). Nous ne pouvons fournir la forme logique de cette phrase, qui contient un pronom qui devrait être traité comme variable liée mais qu'aucun quantificateur restreint ne peut lier. Le second, sémantique, a trait à la croyance conceptuelle attribuée par la seconde phrase de (8). Cette phrase admet une interprétation comme attribution de pensée purement conceptuelle : intuitivement, elle n'engage pas son locuteur à l'existence de fantômes, et peut être vraie sans que les fantômes existent. Malheureusement, nous ne pouvons expliciter la pensée attribuée. En l'absence d'une analyse du pronom, la contribution sémantique de ce dernier nous échappe, et la croyance attribuée par la seconde phrase de (8) demeure évanescente. Pour cette raison, le problème de l'identité intentionnelle souligne notre incompréhension du contenu de certaines croyances attribuées à autrui. Comment saisir la pensée conceptuelle attribuée par la seconde phrase de (8) sans traiter le pronom comme une variable liée, et ce, tout en préservant l'intuition que la valeur sémantique du pronom dépend de celle du quantificateur restreint qui constitue son antécédent?

Comme le note Edelberg ${ }^{16}$, on peut reproduire ce type de problème avec des phrases ne contenant qu'un quantificateur — et pas de quantificateur restreint - comme

(11) Marie croit que quelqu'un hante la maison de Jean et Pierre croit qu'il a brisé des carreaux

(12) Marie croit que quelqu'un hante la maison de Jean et Pierre croit qu'il n'a pas brisé des carreaux

Il va sans dire que le problème se présente aussi pour les phrases plurielles comme

(13) Marie croit que des fantômes hantent la maison de Jean et Pierre croit qu'ils ont brisé des carreaux

I3. Voir Neale, S., Descriptions, The MIT Press, 1990 et Taylor, K., Truth and Meaning, Basil Blackwell, 1998.

I4. Un pronom de paresse ne fait que marquer une place où peut être introduite une expression ayant eu précédemment une occurrence.

I 5. Voir aussi Saarinen, E., "Intentional Identity and Interpretation : A Case Study of the Relations Among Quantifiers, Pronouns and Propositional Attitudes ».

I6. Edelberg, W., " A New Puzzle About Intentional Identity », Journal of Philosophical Logic 15, 1986, p. 1-25 et "Intentional Identity and The Attitudes ", Linguistics and Philosophy 15, 1992, p. 561-596. 
Ce problème peut être généralisé à tous les quantificateurs restreints, maximaux ou non ${ }^{17}$. Ainsi,

(14) Marie croit que quelques fantômes hantent la maison de Jean et Pierre croit qu'ils ont brisé des carreaux

(15) Marie croit que plusieurs fantômes hantent la maison de Jean et Pierre croit qu'ils ont brisé des carreaux

(16) Marie croit que tous les fantômes hantent la maison de Jean et Pierre croit qu'ils ont brisé des carreaux

(17) Marie croit que le fantôme hante la maison de Jean et Pierre croit qu'il a brisé des carreaux

Dans (16) et (17), les pronoms sont apparemment des pronoms de paresse. Je reviendrai sur cette interprétation plausible de ces derniers.

Le fait de ne pouvoir traiter certains pronoms, comme ceux qui nous occupent, ni comme des termes référentiels ni comme des variables liées a certaines conséquences dramatiques pour le défenseur d'une analyse des descriptions (définies et indéfinies) et, plus généralement, des termes quantifiés en termes de quantificateurs restreints. En effet, cette approche semble inapte à saisir des relations évidentes entre ces termes et certains pronoms. À moins de rendre compte de ces relations, force est de porter cette carence à son débit.

J'aimerais ajouter deux détails. Si un pronom est anaphoriquement lié à un quantificateur restreint, alors la valeur sémantique de ce dernier est pertinente pour donner la valeur sémantique du premier. Si la valeur du quantificateur restreint est un contenu conceptuel, alors deux quantificateurs restreints différents fourniront des contenus conceptuels différents. Il semble bien en être ainsi. Comme le note Edelberg ${ }^{18}$, (8) et

(18) Pierre croit qu'un fantôme a brisé des carreaux et Marie croit qu'il hante la maison de Jean

ne sont apparemment pas équivalentes en ce sens que (8) n'implique pas (18), et inversement. (8) semble véhiculer, sémantiquement, que la croyance attribuée à Pierre concerne un fantôme qui hante la maison de Jean, ce qui n'est pas le cas pour celle qui lui est attribuée en (18); (18) semble véhiculer, sémantiquement, que la croyance attribuée à Marie concerne un fantôme qui a brisé des carreaux, ce qui n'est pas le cas pour celle qui lui est attribuée en (8). Une théorie de l'identité intentionnelle devrait rendre compte de cette différence ou, s'il n'y en a pas, de l'illusion de sa présence. En second lieu, étant donné la description du problème en termes d'anaphore et d'attribution de

I7. Un quantificateur restreint de forme $[\mathrm{Dx}: \mathrm{Fx}]$ est maximal si et seulement si une phrase de forme « $[D x: F x](G x)$ » implique une phrase de forme « [Chaque $\mathrm{x}: \mathrm{Fx}](\mathrm{Gx})$ ». Il est non maximal autrement. Dans les exemples qui suivent, (14) et (15) contiennent des quantificateurs restreints non maximaux, alors que (16) et (17) contiennent des quantificateurs restreints maximaux.

I8. Edelberg, W., «A New Puzzle About Intentional Identity ». 
croyance purement conceptuelle, le contenu sémantique fourni par le pronom ne peut refléter que ce qui est précisé par son antécédent.

Revenons à notre exemple initial,

Marie croit qu'un fantôme hante la maison de Jean et Pierre croit qu'il a brisé des carreaux

Dans la description de Saarinen ${ }^{19}$, (8) est utilisée pour dire que Marie et Pierre ont une croyance dirigée vers le même individu, mais ce, non référentiellement. Il me semble plus judicieux de décrire le problème en disant que les deux énoncés d'attitude propositionnelle peuvent être vrais sans qu'existent de fantômes, et surtout que la croyance de Pierre a un contenu similaire à celle de Marie. Si le contenu n'était pas similaire, on ne voit pas comment ces croyances pourraient intuitivement avoir le même foyer.

Les différentes théories présentées pour rendre compte du « il » dans (8) me semblent toutes inadéquates pour diverses raisons. Elles sont $a d$ hoc et pour le moins litigieuses ${ }^{20}$. Je n'argumenterai pas contre celles-ci, et réserverai ce travail pour une autre occasion. Edelberg ${ }^{21}$ a néanmoins présenté une approche séduisante, qui peut paraître naturelle à certains lecteurs.

Edelberg adopte une solution nécessitant la quantification sur modes de présentation et spécifie que Marie et Pierre ont des idées similaires d'un fantôme. C'est là une façon de dire qu'ils pensent au même fantôme. Dans ce contexte, (8) aurait pour forme

$\exists \alpha \exists \beta[\mathrm{B}$ (Marie, [[ $\alpha$ est un fantôme et $\alpha$ hante la maison de Jean]]) et $B$ (Pierre [ $[\beta$ a brisé des carreaux $]]$ ) et $\alpha \approx \beta]$

où $\alpha$ et $\beta$ sont des variables pour idées, et $\approx$ est une relation de contrepartie intentionnelle qui vaut entre deux idées différentes - les idées ne sont pas partagées. J'ai des réserves quant à cette approche. Une théorie qui se dispenserait de la quantification sur des idées serait préférable. D'autant plus que Edelberg ne clarifie ni ce qu'est une idée ni la relation de contrepartie intentionnelle. Par ailleurs, la forme logique assignée à (8) s'éloigne considérablement de sa structure de surface. Une théorie qui donnerait à (8) une forme logique qui s'en éloignerait moins devrait être favorisée. Il y a plus. Si Edelberg préserve certaines intuitions sémantiques, le prix en est la disparition de la relation syntaxique entre le pronom et le quantificateur restreint : rien, dans la forme logique qu'il assigne à (8), ne rend cette relation.

La position que je vais soutenir est générale, s'appuyant sur une théorie ayant des motivations indépendantes. Elle se dispense de la notion d'idée et de

I9. Saarinen, E., « Intentional Identity and Interpretation : A Case Study of the Relations Among Quantifiers, Pronouns and Propositional Attitudes ».

20. Je ne présenterai pas les solutions de Geach, P. («Two Kinds of Intentionality », The Monist 59, 1976, p. 306-320), de Saarinen, E. («Intentional Identity and Interpretation : A Case Study of the Relations Among Quantifiers, Pronouns and Propositional Attitudes »), de Pendlebury, M. ( Hob, Nob and Hecate: The Problem of Quantifying Out ", Australasian Journal of Philosophy 60, $\mathrm{n}^{\circ} .4,1982$, p. 345-354) et de Edelberg, W. ( $\mathrm{A}$ New Puzzle About Intentional Identity ").

2 I. Edelberg, W., "Intentional Identity and The Attitudes ». 
la quantification sur les idées. Elle développe aussi l'intuition que le pronom pertinent, dans les phrases qui nous intéressent, est anaphoriquement lié à un quantificateur restreint. Une théorie qui ne respecterait pas cette intuition exigerait de forts arguments afin de justifier un tel écart.

\section{Les pronoms de texte}

J'ai déjà introduit, afin de rendre compte du comportement sémantique de certains pronoms en ce qui a trait à la référence, la catégorie de pronom de texte ${ }^{22}$. Ces pronoms anaphoriques sont sensibles au quantificateur restreint constituant leur antécédent, en ce sens que la valeur sémantique de ce dernier détermine (partiellement ou totalement) la valeur sémantique - ici, le référent - des premiers, sans être pour autant des variables liées ${ }^{23}$. Par exemple, dans "Le premier astronaute était américain. Il est mort aujourd'hui » et "Un type a téléphoné. Il va rappeler », les deux « il » sont des pronoms de texte. J'ai plus précisément distingué des pronoms de texte de type A, des pronoms ayant pour antécédent des quantificateurs restreints maximaux mais non liés par ces derniers, et des pronoms de texte de type B, des pronoms ayant pour antécédent des quantificateurs restreints non maximaux mais non liés par ceux-ci. J'ai alors formulé des règles de référence pour les pronoms de texte. Les règles de référence d'un pronom de type $B$ sont :

- Le référent sémantique d'un pronom de type B singulier est un objet satisfaisant le contenu descriptif de la description indéfinie antécédente et rendant vraie la phrase contenant la description indéfinie antécédente.

- $\mathrm{Si}$ « a » est un pronom de type B singulier, alors une phrase de forme Fa est vraie si et seulement si un objet ayant la propriété exprimée par la description indéfinie antécédente et rendant vraie la phrase contenant la description indéfinie antécédente est $\mathrm{F}$.

- Le référent sémantique d'un pronom de type B pluriel est constitué par les objets satisfaisant le contenu descriptif du quantificateur restreint antécédent et rendant vraie la phrase contenant le quantificateur restreint antécédent.

- $\mathrm{Si}$ « a » est un pronom de type B pluriel, alors une phrase de forme Fa est vraie si et seulement si tous les objets rendant vraie la phrase contenant le quantificateur restreint antécédent sont $\mathrm{F}$.

Dans notre second exemple, «Un type a téléphoné. Il va rappeler », « il » est un pronom de type $B$, qui désigne un objet, mais un seul, qui est un type et qui a téléphoné. La phrase "Il va rappeler. » est vraie si et seulement si une personne, et une seule, satisfaisant les conditions d'être un type et d'avoir téléphoné, va rappeler.

22. Vallée, R., "Descriptions, référence et anaphore ».

23. Chaque pronom de texte a un et un seul antécédent, qui est le terme dont dépend sa valeur sémantique. 
La règle de référence des pronoms de type $\mathrm{A}$ est la suivante :

- Le référent sémantique d'un pronom de type A est la dénotation russellienne de ce terme.

- $\mathrm{Si}$ « $\mathrm{a}$ » est un pronom de type A, alors une phrase de forme Fa est vraie si et seulement si chaque objet constituant la dénotation russellienne du quantificateur restreint anaphoriquement lié au pronom est $\mathrm{F}$.

Dans notre premier exemple, "Le premier astronaute était américain. Il est mort aujourd'hui », le « il » est un pronom de type A et désigne ce que dénote la description définie qui est son antécédent, en l'occurrence l'unique objet qui est premier astronaute. La phrase «Il est mort aujourd'hui » est vraie si et seulement si l'objet dénoté par « le premier astronaute » est mort aujourd'hui. Les pronoms de type B sont différents des pronoms de type A. Les premiers sont sensibles non seulement au quantificateur restreint qui constitue leur antécédent, mais aussi au prédicat joint à ce terme. Les pronoms de type A ne sont sensibles qu'au quantificateur restreint antécédent. Il va sans dire qu'un locuteur linguistiquement compétent maitrise la sémantique des pronoms de texte, en ce sens qu'il peut identifier les conditions que doit satisfaire le référent du pronom de texte à l'aide des règles de référence formulées plus haut, et connaît les conditions de vérité d'une phrase contenant un tel pronom. Bien entendu, le pronom peut être privé de référent, puisque rien n'assure que les conditions qui lui sont associées soient satisfaites par un objet.

La définition d'un pronom de texte convient parfaitement au pronom « il » ayant une occurrence en (8), de même qu'au pronom «ils» de (14), lesquels seraient des pronoms de type B : ils ont pour antécédent un quantificateur restreint non maximal et ne peuvent être des variables liées par celui-ci à cause, entre autres raisons, de la barrière de la phrase. Mais les pronoms apparaissant dans les clauses indirectes de (8) et de (14) ne réfèrent pas, et sont utilisés afin d'attribuer des contenus conceptuels. Dans la mesure où nous avons besoin d'un contenu conceptuel, des règles de référence semblent inutiles. Nous pouvons cependant construire de tels contenus à partir de ces règles. La règle de référence d'un pronom de type $B$ singulier prescrit que le référent de ce dernier est un objet satisfaisant le quantificateur restreint antécédent et exemplifiant le prédicat qui lui est joint. L'application de la règle, dans un cas particulier, fournit les conditions que doit respecter un objet désigné par ce pronom. Pour notre exemple "Un type a téléphoné. Il va rappeler. », le modèle prédit que le référent de «il » est un type et a téléphoné, où " est un type et a téléphoné » explicite les conditions permettant de déterminer le référent. Les règles fournissent les référents des pronoms de texte par le biais de conditions construites à partir du quantificateur restreint antécédent. Mais ces conditions peuvent aussi servir de contenus de pensée. Il est d'ailleurs plausible de les considérer dans ces termes : il est naturel de penser que le locuteur utilisant la seconde phrase de « Un type a téléphoné. Il va rappeler. » a cognitivement accès à un objet par le biais de ces conditions, qui en constituent le mode de présentation. Traiter les pronoms à l'origine du problème de l'identité intentionnelle comme des pronoms de texte paraît une voie prometteuse afin de rendre compte de nos intuitions sémantiques 
concernant les exemples (8) et (14), et ce, sans recourir à des variables liées. Puisque ces derniers exemples font intervenir des pronoms anaphoriquement liés à des quantificateurs restreints non maximaux ( « un fantôme »), il est judicieux de commencer par l'examen de ceux-ci.

Dans une interprétation en portée large du quantificateur restreint ayant une occurrence dans la première phrase de $(8)$, le pronom ayant une occurrence dans la seconde phrase de (8) ne peut être une variable liée puisque le quantificateur restreint ne traverse pas les limites de la phrase. Dans les circonstances, le pronom qui nous intéresse est naturellement conçu, non comme un terme introduisant un contenu de pensée conceptuel, mais comme un terme référentiel. La seconde phrase de (8) est vraie si et seulement si Pierre croit qu'un fantôme particulier — celui désigné par «il »- a brisé des carreaux. On peut aisément traiter ce pronom comme un pronom de texte. Considérons (8) dans une interprétation du quantificateur restreint " un fantôme » en portée large, pour avoir

[Un x: fantôme $\mathrm{x}$ ] Marie croit que ( $\mathrm{x}$ hante la maison de Jean) et Pierre croit qu'il a brisé des carreaux

Le « il » est alors intuitivement un pronom de texte de type B, et désigne un fantôme, mais un seul, qui rend vraie la phrase enchâssée dans le terme d'attitude propositionnelle de la première phrase de (8). Si on examine (8) en donnant une portée large au quantificateur restreint, et si « il » est un pronom de texte, la règle prescrit que le référent de ce dernier est donné par « est un fantôme et hante la maison de Jean ». Puisque les fantômes n'existent pas, le pronom ne désigne rien. La croyance attribuée à Pierre devrait contenir le référent du pronom de texte. Puisque ce pronom ne désigne rien, Pierre se voit attribuer une pensée incomplète.

Considérons maintenant (8) en donnant au quantificateur restreint « un fantôme » une portée étroite. On peut utiliser le résultat de l'application de la règle fournissant les conditions déterminant le référent du pronom comme contenu de la croyance attribuée par la seconde phrase de (8). On obtient alors

(20) Marie croit qu'un fantôme hante la maison de Jean et Pierre croit qu'un objet qui est un fantôme et qui hante la maison de Jean a brisé des carreaux

Il est aisé de donner à (20) une forme logique satisfaisant nos intuitions linguistiques concernant le problème de l'identité intentionnelle. On obtient alors « Marie croit que ([un x: fantôme $\mathrm{x}]$ (x hante la maison de Jean)) et Pierre croit que ([un $\mathrm{x}$ : fantôme $\mathrm{x}$ et hante la maison de Jean $\mathrm{x}$ ] ( $\mathrm{x}$ a brisé des carreaux)). » Une expression de forme « un objet qui est $F$ est $G$ » est vraie si et seulement si $|F-G|=0$, soit si et seulement si l'intersection des $F-$ ici un objet qui est un fantôme et qui hante la maison de Jean — et des non $\mathrm{G}$ - des objets qui n'ont pas la propriété d'avoir brisé des carreaux — est vide. Le nombre du pronom, le singulier, contraint à une interprétation singulière de « un objet qui 
est un fantôme et qui hante la maison de Jean », soit $|\mathrm{F}|=1$, et spécifie donc qu'un seul objet qui satisfait des conditions d'être un fantôme et de hanter la maison de Jean a brisé des carreaux. La relation d'anaphore est saisie par la règle générale, qui prescrit que la valeur sémantique du pronom de texte est partiellement fonction de son antécédent et, dans chaque cas particulier, elle donne des résultats différents. Il est important de souligner que cette approche ne signifie pas que le contenu est anaphorique, puisque les pronoms, et non les contenus, sont anaphoriques. Pierre pourrait aussi avoir le contenu conceptuel qui lui est attribué, et ce, indépendamment de ce que croit Marie. On peut aisément généraliser ce modèle à tous les pronoms de textes, en s'appuyant sur le nombre du pronom afin de rendre compte de la différence entre le pluriel et le singulier. Pour les phrases contenant les pronoms de type B pluriels

(10) Marie croit que des fantômes hantent la maison de Jean et Pierre croit qu'ils ont brisé des carreaux

(11) Marie croit que quelques fantômes hantent la maison de Jean et Pierre croit qu'ils ont brisé des carreaux

(12) Marie croit que plusieurs fantômes hantent la maison de Jean et Pierre croit qu'ils ont brisé des carreaux

on obtient

(21) Marie croit que des fantômes hantent la maison de Jean et Pierre croit que des objets qui sont des fantômes et qui hantent la maison de Jean ont brisé des carreaux

(22) Marie croit que quelques fantômes hantent la maison de Jean et Pierre croit que des objets qui sont des fantômes et qui hantent la maison de Jean ont brisé des carreaux

(23) Marie croit que plusieurs fantômes hantent la maison de Jean et Pierre croit que des objets qui sont des fantômes et qui hantent la maison de Jean ont brisé des carreaux

où, dans chacun des cas, le pluriel du pronom impose une interprétation plurielle du contenu, pour obtenir « sont des objets qui sont des fantômes et hantent la maison de Jean ».

On pourrait émettre des réserves quant à l'insertion, dans la seconde phrase de chacun des exemples précédent, de l'information fournie par le prédicat de la première phrase en contexte d'attitude propositionnelle ${ }^{24}$. Il est pourtant juste d'ajouter cette information. Si le pronom «il » faisait écho uniquement au contenu conceptuel du quantificateur restreint, nous obtiendrions des résultats inacceptables. Supposons que Marie et Pierre n'aient aucun fantôme particulier à l'esprit, mais simplement des croyances purement conceptuelles. Si « il » reflète simplement le contenu conceptuel de " un fantôme ", alors la phrase " Marie croit qu'un fantôme hante la maison de Jean et Pierre croit qu'il ne hante pas la maison de Jean. » devrait être

24. Soulignons au passage que Pendlebury ("Hob, Nob and Hecate: The Problem of Quantifying Out", p. 350) semble partager mes intuitions linguistiques. 
acceptable. Mais elle est bizarre, sinon contradictoire. Qui plus est, contrairement à la caractérisation de l'identité intentionnelle fournie par Geach, les deux personnes auxquelles sont faites les attributions n'ont alors pas, intuitivement, des croyances ayant le même foyer. Le lecteur peut mettre ses intuitions linguistiques à l'épreuve en considérant les attributions de croyance conceptuelle " Marie croit qu'un fantôme hante la maison de Jean et Pierre croit que le même fantôme ne hante pas la maison de Jean. ". Cette incongruité est expliquée si on soutient que l'information du prédicat qui nous concerne, et qui devrait se trouver dans la phrase dans le second contexte d'attitude propositionnelle, est alors niée. À moins qu'un fantôme particulier soit en question, lequel serait désigné par le "il » de (8), il est impossible d'interpréter la seconde phrase de (8). Et si elle est ainsi interprétée, elle n'est pas utilisée pour attribuer une croyance conceptuelle.

Portons maintenant attention aux pronoms anaphoriques avec des quantificateurs restreints maximaux, soit les exemples (16) et (17). Une analyse en termes de pronom de paresse est possible. Mais une autre s'appuyant sur la sémantique des pronoms de type A l'est aussi, et cette dernière permet une intéressante généralisation. Je crois préférable d'aborder les pronoms qui nous intéressent en termes de pronom de type A, même si les résultats sont équivalents à une analyse en termes de pronom de paresse, parce que les pronoms de type A rendent compte des pronoms que nous examinons et en expliquent le fonctionnement sémantique, alors que les qualifier de pronoms de paresse ne me semble que répéter nos intuitions, sans les expliquer. Considérons :

(16) Marie croit que tous les fantômes hantent la maison de Jean et Pierre croit qu'ils ont brisé des carreaux

(17) Marie croit que le fantôme hante la maison de Jean et Pierre croit qu'il a brisé des carreaux

En s'appuyant sur la sémantique du pronom comme pronom de type A, on peut rendre ces exemples par, respectivement,

(24) Marie croit que tous les fantômes hantent la maison de Jean et Pierre croit que tous les fantômes ont brisé des carreaux

(25) Marie croit que le fantôme hante la maison de Jean et Pierre croit que le fantôme a brisé des carreaux

Ces résultats sont fidèles à nos intuitions et les expliquent à l'intérieur d'un cadre sémantique général. En ce sens, ma suggestion est préférable à une explication en termes de pronom de paresse. Cette dernière produit une analyse conduisant aux mêmes conclusions, sans en donner de justification. 
Il devrait être évident que mon approche capture l'intuition que (8) et (18) ne sont pas équivalentes. Les croyances attribuées en (8) sont données par (20), tandis que celles attribuées en (18) sont données par

(26) Pierre croit qu'un fantôme a cassé des carreaux et Marie croit qu'un objet qui est un fantôme et qui a cassé des carreaux hante la maison de Jean

\section{Deux objections}

Considérons maintenant la phrase

(27) Marie croit qu'un étudiant est venu à 2 heures et Pierre croit qu'il est venu à 4 heures

en spécifiant que (27) attribue une croyance conceptuelle à Marie et une autre à Pierre. Mais quelle croyance conceptuelle est attribuée à Pierre en (27)? Selon le modèle que je propose, si le quantificateur restreint " un étudiant " a une portée étroite et si le pronom de la seconde phrase est anaphoriquement lié à celui-ci, (27) implique que Pierre croit qu'un étudiant qui est venu à 2 heures est venu à 4 heures. Dans l'interprétation de (27) comme exemple d'identité intentionnelle, la seconde phrase de (27) ne constitue donc pas une attribution de croyance conceptuelle cohérente ${ }^{25}$. Le pronom de (27) ne peut donc être conçu comme un pronom de texte utilisé pour attribuer une pensée conceptuelle ayant le même foyer que celle attribuée à Marie. Le locuteur de la seconde phrase de (27) a alors plausiblement à l'esprit un certain étudiant et le pronom « il » réfère à celui-ci. La seconde phrase de (27) n'est alors pas une attribution de croyance conceptuelle. Revenons enfin à une interprétation de (8) mentionnée au début de ce texte, laquelle est, je le rappelle, différente de celle qui nous intéresse et qui fut justifiée lors de la présentation de notre problème : la seconde phrase de (27) peut signifier que Pierre croit que l'étudiant que Marie croit être venu à 2 heures est venu à 4 heures. Cette interprétation est plausible. Mais comment la construire à partir du « il »? Comment peut-elle être récupérée sur la base de la syntaxe et la sémantique de ce pronom? Si elle est pragmatiquement donnée, quels sont les mécanismes mis en œuvre pour la récupérer? Je ne puis, faute d'espace, approfondir ces questions.

\section{Conclusion}

Si les pronoms comme celui que l'on retrouve dans la seconde phrase de (8) sont des pronoms de texte, alors on peut rendre compte de l'identité intentionnelle, et ce, sans qu'il soit nécessaire de quantifier à l'extérieur. On pourrait m'objecter que Pierre, dans l'exemple (8), n'a pas le contenu mental qui lui est attribué en (20) parce qu'il ne croit pas qu'un fantôme hante la maison de Jean. Dans ces conditions, le pronom ne saurait être considéré comme anaphoriquement lié au quantificateur restreint antécédent : il ne

25. Si Pierre croit qu'il est revenu à 4 heures, le problème ne se pose pas. 
peut être une variable liée, et le faire dépendre de la valeur sémantique de " un fantôme » ne donne pas des résultats satisfaisants. Le défenseur de cette position devra alors expliquer comment saisir nos intuitions que Marie et Jean ont des croyances purement conceptuelles ayant le même foyer et ce, sans que la valeur sémantique du pronom dépende de celle du quantificateur restreint antécédent. Rendre compte de la présence d'un même foyer sans tenir compte de la relation d'anaphore est non seulement désespéré, mais aussi discutable d'un point de vue théorique : il y a une évidente relation entre le pronom et le quantificateur restreint antécédent, et nier l'existence de cette relation exige au moins un argument. S'offrent deux possibilités : soit que la seconde phrase de (8) ait un contenu conceptuel qu'il est possible de spécifier à l'aide de (8), soit qu'il en ait un qu'il impossible de spécifier à l'aide de cette seule ressource. Dans le premier cas, aucun pronom anaphorique ne saurait récupérer plus, ou moins, de détails que ceux fournis par les pronoms de texte. Un défenseur de cette approche devrait alors nous expliquer quelle ressources il met à profit afin d'expliciter la croyance attribuée. Dans le second cas, le pronom ne serait tout simplement pas anaphoriquement lié au quantificateur restreint antécédent. Un défenseur de cette dernière approche nous devrait une explication de la relation entre le terme quantifié et le pronom, et surtout une théorie permettant d'expliciter le contenu conceptuel attribué par la seconde phrase de (8). 